\title{
Commutators with Lipschitz Functions and Nonintegral Operators
}

\author{
Peizhu Xie ${ }^{1,2}$ and Ruming Gong ${ }^{1,2}$ \\ ${ }^{1}$ School of Mathematics and Information Science, Guangzhou University, Guangzhou 510006, China \\ ${ }^{2}$ Key Laboratory of Mathematics and Interdisciplinary Sciences of Guangdong Higher Education Institutes, \\ Guangzhou University, Guangzhou 510006, China
}

Correspondence should be addressed to Peizhu Xie; xiepeizhu82@163.com

Received 29 March 2013; Accepted 30 May 2013

Academic Editor: Alberto Cabada

Copyright (C) 2013 P. Xie and R. Gong. This is an open access article distributed under the Creative Commons Attribution License, which permits unrestricted use, distribution, and reproduction in any medium, provided the original work is properly cited.

Let $T$ be a singular nonintegral operator; that is, it does not have an integral representation by a kernel with size estimates, even rough. In this paper, we consider the boundedness of commutators with $T$ and Lipschitz functions. Applications include spectral multipliers of self-adjoint, positive operators, Riesz transforms of second-order divergence form operators, and fractional power of elliptic operators.

\section{Introduction}

Let $T$ be a bounded operator on $L^{p}\left(\mathbb{R}^{n}\right)$ for some $p, 1<$ $p<\infty$. A measurable function $K(x, y)$ is called an associated kernel of $T$ if

$$
T f(x)=\int_{X} K(x, y) f(y) d y
$$

holds for each continuous function $f$ with compact support and for almost all $x$ not in the support of $f$.

The kernel $K(x, y)$ is said to satisfy the following.

(i) The pointwise Hörmander condition on $x$ variable if there exist $0<\alpha \leq 1$ and $c, c_{1} \geq 1$ such that

$$
|K(x, y)-K(z, y)| \leq c \frac{|x-z|^{\alpha}}{|x-y|^{n+\alpha}},
$$

when $|x-y| \geq c_{1}|x-z|$, and $B(x, r)$ denotes the ball with center $x$, radius $r$.

(ii) The integral Hörmander condition on $y$ variable if there exist constants $C$ and $c_{2} \geq 1$ such that

$$
\int_{|x-y| \geq c_{2}|z-y|}|K(x, y)-K(x, z)| d x \leq C,
$$

for all $y, z \in \mathbb{R}^{n}$.
It is well known that if $T$ is bounded on $L^{q}\left(\mathbb{R}^{n}\right)$ for some $q, 1<q<\infty$, and $b \in \mathrm{BMO}$, the two Hörmander conditions (i) and (ii) above are sufficient to imply that the commutator $[b, T]$ is bounded on $L^{p}\left(\mathbb{R}^{n}\right)$ for all $p, 1<p<\infty$, with norm

$$
\|[b, T](f)\|_{p} \leq C\|b\|_{*}\|f\|_{p},
$$

where the commutator $[b, T]$ is defined by $[b, T](f)=T(b f)-$ $b T(f)$ and $\|b\|_{*}$ is the BMO seminorm of $b$. See $[1,2]$ for BMO functions on Euclidean spaces $\mathbb{R}^{n}$ and [3] for spaces of homogeneous type.

A particular case of the result of Janson [2] states that $[b, T]: L^{p} \rightarrow L^{q}$ is bounded, $1<p<q<\infty$, if $b \in \dot{\Lambda}_{\beta}$, $\beta=n(1 / p-1 / q)$. Here, $\dot{\Lambda}_{\beta}$ is the homogeneous Lipschitz space determined by the first difference operator.

In [4], Duong and Yan have replaced the two Hörmander conditions (2) and (3) by the following weaker conditions (5) and (6) below which previously appeared in [5] and still concluded that the commutator $[b, T]$ is bounded on $L^{p}\left(\mathbb{R}^{n}\right)$ for all $p, 1<p<\infty$. And in [6], Hu and Yang obtained the weighted boundedness of maximal commutator when $T$ satisfy (5) and (6). Roughly speaking, we assume the following.

(iii) There exists a class of operators $A_{t}$ with kernels $a_{t}(x, y)$, which satisfy the condition (23) in Section 2 , so that 
the kernels $k_{t}(x, y)$ of the operators $\left(T-A_{t} T\right)$ satisfy the condition

$$
\left|k_{t}(x, y)\right| \leq c \frac{t^{\gamma / m}}{|x-y|^{n+\gamma}}
$$

when $|x-y| \geq c_{2} t^{1 / m}$ for some $\gamma, m>0$, where $c$ is a positive constant.

(iv) There exists a class of operators $B_{t}$ with kernels $b_{t}(x, y)$, which satisfy the condition $(23)$, such that $(T-$ $\left.T B_{t}\right)$ have associated kernels $K_{t}(x, y)$ and there exist positive constants $c_{3}, c_{4}$ such that

$$
\int_{|x-y| \geq c_{3} t^{1 / m}}\left|K_{t}(x, y)\right| d x \leq c_{4}, \quad \forall y \in \mathbb{R}^{n} .
$$

Under conditions (5) and (6), if $T$ is bounded on $L^{q}\left(\mathbb{R}^{n}\right)$ for some $q, 1<q<\infty$, then the commutator $[b, T]$ is bounded on $L^{p}\left(\mathbb{R}^{n}\right)$ for all $p, 1<p<\infty$.

In [7], Auscher and Martell have considered the commutators of singular nonintegral operators, where the implicit terminology has been introduced in [8]. By this we mean that they are still of order 0 , but they do not have an integral representation by a kernel with size and/or smoothness estimates. Let $1 \leq p_{0}<q_{0} \leq \infty$. Suppose that the singular nonintegral operator $T$ is a sublinear operator bounded on $L^{p_{0}}\left(\mathbb{R}^{n}\right)$ and that $\left\{A_{r}\right\}_{r>0}$ is a family of operators acting from $L_{c}^{\infty}\left(\mathbb{R}^{n}\right)$ into $L^{p_{0}}\left(\mathbb{R}^{n}\right)$. Auscher and Martell assume the following.

(v) For all $f \in L_{c}^{\infty}\left(\mathbb{R}^{n}\right)$ and all balls $B$ where $r(B)$ denotes its radius,

$$
\begin{aligned}
& \left(\frac{1}{|B|} \int_{B}\left|T\left(I-A_{r(B)}\right) f\right|^{p_{0}} d x\right)^{1 / p_{0}} \\
& \quad \leq C \sum_{j=1}^{\infty} \alpha_{j}\left(\frac{1}{\left|2^{j+1} B\right|} \int_{2^{j+1} B}|f|^{p_{0}} d x\right)^{1 / p_{0}} .
\end{aligned}
$$

(vi) For all $f \in L_{c}^{\infty}\left(\mathbb{R}^{n}\right)$ and all balls $B$ where $r(B)$ denotes its radius,

$$
\begin{aligned}
& \left(\frac{1}{|B|} \int_{B}\left|T A_{r(B)} f\right|^{q_{0}} d x\right)^{1 / q_{0}} \\
& \quad \leq C \sum_{j=1}^{\infty} \alpha_{j}\left(\frac{1}{\left|2^{j+1} B\right|} \int_{2^{j+1} B}|T f|^{p_{0}} d x\right)^{1 / p_{0}}
\end{aligned}
$$

Let $p_{0}<p<q_{0}$ and $w \in A_{p / p_{0}} \cap R H_{\left(q_{0} / p\right)^{\prime}}$ (for the definitions of $A_{p / p_{0}}$ and $R H_{\left(q_{0} / p\right)^{\prime}}$ see Section 2). Under conditions (7) and (8), if $\sum_{j=1}^{\infty} \alpha_{j} j<\infty$, then the commutator $[b, T]$ is bounded on $L^{p}(w)$; that is, $\|[b, T] f\|_{L^{p}(w)} \leq C\|b\|_{*}\|f\|_{L^{p}(w)}$ for all $f \in L_{c}^{\infty}\left(\mathbb{R}^{n}\right)$.

The main object of this paper is the commutators of nonintegral operators $[b, T]$. Compared to the result in [7], we can obtain a more general result for $b$ belongs to the Lipschitz spaces $\dot{\Lambda}_{\beta_{i}}(X)$. To be more specific, we can obtain the following.

Theorem 1. Let $0 \leq \alpha<1,1 \leq p_{0} \leq s_{0}<q_{0} \leq \infty$ such that $1 / s_{0}=1 / p_{0}-\alpha / n$. Suppose that $T$ is a sublinear operator bounded from $L^{p_{0}}\left(\mathbb{R}^{n}\right)$ to $L^{s_{0}}\left(\mathbb{R}^{n}\right)$ and that $\left\{A_{r}\right\}_{r>0}$ is a family of operators acting from $L_{c}^{\infty}\left(\mathbb{R}^{n}\right)$ into $L^{p_{0}}\left(\mathbb{R}^{n}\right)$. Assume that

$$
\begin{aligned}
& \left(\frac{1}{|B|} \int_{B}\left|T\left(I-A_{r(B)}\right) f\right|^{s_{0}} d x\right)^{1 / s_{0}} \\
& \quad \leq C \sum_{j=1}^{\infty} \alpha_{j}\left|2^{j+1} B\right|^{\alpha / n}\left(\frac{1}{\left|2^{j+1} B\right|} \int_{2^{j+1} B}|f|^{p_{0}} d x\right)^{1 / p_{0}} \\
& \left(\frac{1}{|B|} \int_{B}\left|T A_{r(B)} f\right|^{q_{0}} d x\right)^{1 / q_{0}} \\
& \quad \leq C \sum_{j=1}^{\infty} \alpha_{j}\left(\frac{1}{\left|2^{j+1} B\right|} \int_{2^{j+1} B}|T f|^{s_{0}} d x\right)^{1 / s_{0}}
\end{aligned}
$$

for all $f \in L_{c}^{\infty}\left(\mathbb{R}^{n}\right)$ and all balls $B$, where $r(B)$ denotes its radius. Let $0<\beta<1$ such that $\alpha+\beta<1$. Let $p_{0}<p<q<q_{0}$ and $1 / q=1 / p-(\alpha+\beta) / n$. If $\sum_{j=1}^{\infty} \alpha_{j}<\infty$, then there is $a$ constant $C$ such that

$$
\|[b, T] f\|_{L^{q}} \leq C\|b\|_{\Lambda_{\beta}}\|f\|_{L^{p}}
$$

for all $f \in L_{c}^{\infty}\left(\mathbb{R}^{n}\right)$ and for all $b \in \dot{\Lambda}_{\beta}$.

The case $q_{0}=\infty$ is understood in the sense that the $L^{q_{0}}$ average in (10) is indeed an essential supremum.

Remark 2. Let $1 \leq p_{0}<p<q<q_{0}$ be such that $1 / q=1 / p-$ $\alpha / n$. Under the assumptions above, we know that if $\sum_{j=1}^{\infty} \alpha_{j}<$ $\infty$, then $T$ is bounded from $L^{p}$ to $L^{q}$. See Theorem 2.2 in [9].

In the limiting case $\alpha=0$, from the assumptions (9) and (10), we deduce

$$
\begin{gathered}
\left(\frac{1}{|B|} \int_{B}\left|T\left(I-A_{r(B)}\right) f\right|^{p_{0}}\right)^{1 / p_{0}} \leq C M\left(|f|^{p_{0}}\right)^{1 / p_{0}}(x) \\
\left(\frac{1}{|B|} \int_{B}\left|T A_{r(B)} f\right|^{q_{0}}\right)^{1 / q_{0}} \leq C M\left(|T f|^{p_{0}}\right)^{1 / p_{0}}(x) .
\end{gathered}
$$

Consequently, from the Theorem 3.7 in [7], we know that if $\sum_{j=1}^{\infty} \alpha_{j}<\infty$, then $\|T f\|_{L^{p}(w)} \leq C\|f\|_{L^{p}(w)}$ for $p_{0}<p<q_{0}$ and for all $w \in A_{p / p_{0}} \cap R H_{\left(q_{0} / p\right)^{\prime}}$.

Theorem 3. Let $1 \leq p_{0}<q_{0} \leq \infty$. Suppose that $T$ is a sublinear operator bounded on $L^{P_{0}}\left(\mathbb{R}^{n}\right)$ and that $\left\{A_{r}\right\}_{r>0}$ is a family of operators acting from $L_{c}^{\infty}\left(\mathbb{R}^{n}\right)$ to $L^{P_{0}}\left(\mathbb{R}^{n}\right)$. Assume that $T$ satisfy (9) and (10) with $\alpha=0$. Let $0<\beta<$ $\min \left\{1, n / p_{0}\right\}, p_{0}<p<q<q_{0}, b \in \dot{\Lambda}_{\beta}$ and $w, v \in$ $A_{p / p_{0}} \cap R H_{\left(q_{0} / p\right)^{\prime}}$. Assume that there exists a constant $1<s<$ $\min \left\{n / \beta p_{0}, p / p_{0}\right\}$ such that $(w, v) \in A\left(p / p_{0} s, q / p_{0} s, \beta p_{0} s / n\right)$. If $\sum_{j=1}^{\infty} \alpha_{j}<\infty$, then there is a constant $C$ such that

$$
\|[b, T] f\|_{L^{q}(v)} \leq C\|b\|_{\dot{\Lambda}_{\beta}}\|f\|_{L^{p}(w)},
$$

for all $f \in L_{c}^{\infty}$.

The class $A(p, q, s)$ is defined in Section 2. 


\section{Definitions and Preliminary Results}

We use the notation

$$
f_{E} f=\frac{1}{|E|} \int_{E} f(x) d x
$$

and we often ignore the Lebesgue measure and the variable of the integrand in writing integrals, unless this is needed to avoid confusions.

A weight $w$ is a nonnegative locally integrable function. We say that $w \in A_{p}, 1<p<\infty$, if there exists a constant $C$ such that for every ball $B \subset X$

$$
\left(f_{B} w\right)\left(f_{B} w^{1-p^{\prime}}\right)^{p-1} \leq C .
$$

For $p=1$, we say that $w \in A_{1}$ if there is a constant $C$ such that for every ball $B \subset \mathbb{R}^{n}, f_{B} w \leq C w(x)$, for a.e. $x \in$ $B$, or, equivalently, $M(w) \leq C w$ a.e., where $M(w)$ denotes the classical Hardy-Littlewood maximal function of $w$. The reverse Hölder classes are defined in the following way: $w \in$ $R H_{q}, 1<q<\infty$, if there is a constant $C$ such that for every ball $B \subset \mathbb{R}^{n}$

$$
\left(f_{B} w^{q}\right)^{1 / q} \leq f_{B} w .
$$

The endpoint $q=\infty$ is given by the condition: $w \in R H_{\infty}$ whenever, for any ball $B$,

$$
w(x) \leq f_{B} w, \quad \text { for a.e. } x \in B
$$

The homogenous Lipschitz function space $\dot{\Lambda}_{\beta}\left(\mathbb{R}^{n}\right)$ is the space of functions $f$ such that

$$
\|f\|_{\dot{\Lambda}_{\beta}}=\sup _{x, h \in \mathbb{R}^{n}, h \neq 0} \frac{\left|\Delta_{h}^{[\beta]+1} f(x)\right|}{|h|^{\beta}}<\infty,
$$

where $\Delta_{h}^{k}$ denotes the $k$ th difference operator (see [10]). That is, $\Delta_{h}^{1} f(x)=\Delta_{h} f(x)=f(x+h)-f(x), \Delta_{h}^{k+1} f(x)=\Delta_{h}^{k} f(x+$ h) $-\Delta_{h}^{k} f(x), k \geq 1$.

We have the following lemmas.

Lemma 4 (see [10]). For $0<\beta<1,1 \leq q<\infty$, one has

$$
\begin{aligned}
\|f\|_{\dot{\Lambda}_{\beta}\left(\mathbb{R}^{n}\right)} & \approx \sup _{B} \frac{1}{|B|^{1+\beta / n}} \int_{B}\left|f-f_{B}\right| d x \\
& \approx \sup _{B} \frac{1}{|B|^{\beta / n}}\left(\frac{1}{|B|} \int_{B}\left|f-f_{B}\right|^{q}\right)^{1 / q} d x .
\end{aligned}
$$

For $q=\infty$, the last formula should be modified appropriately.

Lemma 5 (see $[10])$. Let $B^{*} \subset B \subset \mathbb{R}^{n}$, and then $\left|f_{B^{*}}-f_{B}\right| \leq$ $C\|f\|_{\dot{\Lambda}_{\beta}\left(\mathbb{R}^{n}\right)}|B|^{\beta / n}$.

Lemma 6 (see [11]). For $1 \leq \gamma<\infty$ and $\beta>0$, let

$$
M_{\beta, \gamma}(f)(x)=\sup _{B \ni x}\left(\frac{1}{|B|^{1-\beta \gamma / n}} \int_{B}|f(y)|^{\gamma} d y\right)^{1 / \gamma} .
$$

Suppose that $\gamma<p<n / \beta$ and $1 / q=1 / p-\beta / n$, and then

$$
\left\|M_{\beta, \gamma}(f)\right\|_{L^{q}\left(\mathbb{R}^{n}\right)} \leq C\|f\|_{L^{p}\left(\mathbb{R}^{n}\right)^{*}} .
$$

Theorem A (see [7]). Fix $1<q \leq \infty, a \geq 1$, and $\omega \in R H_{s^{\prime}}$, $1 \leq s<\infty$. Then, there exist $C=C(q, n, a, \omega, s)$ and $K_{0}=$ $K_{0}(n, a) \geq 1$ with the following property: assume that $F, G$, $H_{1}$, and $H_{2}$ are nonnegative measurable functions on $\mathbb{R}^{n}$ such that for any cube $Q$ there exist nonnegative functions $G_{Q}$ and $H_{\mathrm{Q}}$ with $F(x) \leq G_{\mathrm{Q}}(x)+H_{\mathrm{Q}}(x)$ for a.e. $x \in Q$ and

$$
\begin{gathered}
\left(f_{Q} H_{Q}^{q}\right)^{1 / q} \leq a\left(M F(x)+M H_{1}(x)+H_{2}(\bar{x})\right), \\
\forall x, \bar{x} \in Q, \\
f_{Q} G_{Q} \leq G(x), \quad \forall x \in Q .
\end{gathered}
$$

Then for all $\lambda>0, K \geq K_{0}$ and $0<\gamma<1$

$$
\begin{aligned}
\omega\{M F & \left.>K \lambda, G+H_{2} \leq \gamma \lambda\right\} \\
& \leq C\left(\frac{a^{q}}{K^{q}}+\frac{\gamma}{K}\right)^{1 / s} \omega\left\{M F+M H_{1}>\lambda\right\} .
\end{aligned}
$$

As a consequence, for all $0<p<1 / s$, one has

$$
\begin{aligned}
& \|M F\|_{L^{p}(\omega)} \\
& \quad \leq C\left(\|G\|_{L^{p}(\omega)}+\left\|M H_{1}\right\|_{L^{p}(\omega)}+\left\|H_{2}\right\|_{L^{p}(\omega)}\right),
\end{aligned}
$$

provided $\|M F\|_{L^{p}(\omega)}<\infty$, and

$$
\begin{aligned}
& \|M F\|_{L^{p, \infty}(\omega)} \\
& \quad \leq C\left(\|G\|_{L^{p, \infty}(\omega)}+\left\|M H_{1}\right\|_{L^{p, \infty}(\omega)}+\left\|H_{2}\right\|_{L^{p, \infty}(\omega)}\right),
\end{aligned}
$$

provided $\|M F\|_{L^{p, \infty}(\omega)}<\infty$. Furthermore, if $p \geq 1$, then (24) and (25) hold, provided $F \in L^{1}$ (whether or not $M F \in L^{p}(\omega)$ ).

For $0<s<1$ and $1 \leq \gamma<\infty$, we denote

$$
\mathscr{M}_{s, \gamma}(f)(x)=\sup _{B \ni x}\left(\frac{1}{|B|^{1-s}} \int_{B}|f(y)|^{\gamma} d y\right)^{1 / \gamma},
$$

where the supremum is taken with respect to all balls $B$ of positive measure containing the point $x$.

Theorem B. Let $1<p<q<\infty, 0<s<1$, and let $v$ and $w$ be the weight functions. For a constant $C>0$ to exist so that the inequality

$$
\begin{gathered}
\left(\int_{\mathbb{R}^{n}}\left(\mathscr{M}_{s, 1}(f)(x)\right)^{q} v(x) d x\right)^{1 / q} \\
\quad \leq C\left(\int_{\mathbb{R}^{n}}|f(x)|^{p} w(x) d x\right)^{1 / p}
\end{gathered}
$$


would hold, it is necessary and sufficient that the condition

$$
\begin{aligned}
& \sup _{x \in \mathbb{R}^{n}, r>0}\left(w^{1-p^{\prime}} B(x, 6 r)\right)^{1 / p^{\prime}} \\
& \quad \times\left(\int_{\mathbb{R}^{n} \backslash B(x, r)} v(y)|x-y|^{(s-1) q n} d y\right)^{1 / q}<\infty,
\end{aligned}
$$

where $1 / p+1 / p^{\prime}=1$, be fulfilled.

For the proof of this theorem, see [12].

Definition 7. $(w, v)$ is said to belong to $A(p, q, s)(1<p<q<$ $\infty, 0<s<1)$ if $(28)$ holds.

Lemma 8. Let $1 \leq \gamma<p<q<\infty, 0<s<1$. If $(w, v) \in$ $A(p / \gamma, q / \gamma, s)$, then

$$
\left\|\mathscr{M}_{s, \gamma} f\right\|_{L^{q}(v)} \leq C\|f\|_{L^{p}(w)} .
$$

Proof. Since $\mathscr{M}_{s, \gamma}(f)(x)=\left(\mathscr{M}_{s, 1}\left(|f|^{\gamma}\right)(x)\right)^{1 / \gamma}$, we have

$$
\begin{aligned}
\left\|\mathscr{M}_{s, \gamma} f\right\|_{L^{q}(v)} & =\left\|\left(\mathscr{M}_{s, 1}\left(|f|^{\gamma}\right)\right)^{1 / \gamma}\right\|_{L^{q}(v)} \\
& =\left\|\mathscr{M}_{s, 1}\left(|f|^{\gamma}\right)\right\|_{L^{q / \gamma}(v)}^{1 / \gamma} .
\end{aligned}
$$

By Theorem B, we have

$$
\begin{aligned}
\left\|\mathscr{M}_{s, 1}\left(|f|^{\gamma}\right)\right\|_{L^{q / \gamma}(v)} & \leq C\left\||f|^{\gamma}\right\|_{L^{p / \gamma}(w)} \\
& =C\|f\|_{L^{p}(w)}^{\gamma} .
\end{aligned}
$$

Thus,

$$
\left\|\mathscr{M}_{s, \gamma} f\right\|_{L^{q}(v)} \leq C\|f\|_{L^{p}(w)} .
$$

\section{The Proof of the Main Theorems}

In order to prove Theorem 1, we need the following lemma.

Lemma 9. Let $1 \leq p_{0} \leq s_{0}, p_{0}<p<q<\infty$, and $w, v \in A_{\infty}$. Let $T$ be a sublinear operator bounded from $L^{p_{0}}$ to $L^{s_{0}}$.

(i) If $b \in \dot{\Lambda}_{\beta} \cap L^{\infty}$ and $f \in L_{c}^{\infty}$, then $[b, T] f \in L^{s_{0}}$.

(ii) Assume that for any $b \in \dot{\Lambda}_{\beta} \cap L^{\infty}$ and for any $f \in L_{c}^{\infty}$ one has that

$$
\|[b, T] f\|_{L^{q}(v)} \leq C\|b\|_{\dot{\Lambda}_{\beta}}\|f\|_{L^{p}(w)},
$$

where $C$ does not depend on $b$ and $f$. Then for all $b \in \dot{\Lambda}_{\beta}$, holds.

Proof. The ideas of the following argument are taken from [7]. Fix $f \in L_{c}^{\infty}$. Note that (i) follows easily observing that

$$
\begin{aligned}
{[b, T] f(x) } & \leq|b(x)||T f(x)|+|T(b f)(x)| \\
& \leq\|b\|_{L^{\infty}}|T f(x)|+|T(b f)(x)| \in L^{s_{0}}
\end{aligned}
$$

since $b \in L^{\infty}, f \in L_{c}^{\infty}$ imply that $f, b f \in L_{c}^{\infty} \subset L^{p_{0}}$ and hence, by assumption, $T(f), T(b f) \in L^{s_{0}}$.

To obtain (ii), we fix $b \in \dot{\Lambda}_{\beta}$ and $f \in L_{c}^{\infty}$. Let $Q_{0}$ be a cube such that $\operatorname{supp} f \subset Q_{0}$. We may assume that $b_{\mathrm{Q}_{0}}=0$ since otherwise we can work with $\bar{b}=b-b_{\mathrm{Q}_{0}}$ and observe that

$$
[b, T]=[\bar{b}, T], \quad\|b\|_{\dot{\Lambda}_{\beta}}=\|\bar{b}\|_{\dot{\Lambda}_{\beta}} .
$$

Note that for $m=0,1$, we have that $\left|b^{m} f\right|$ and $\left|T\left(b^{m} f\right)\right|$ are finite almost everywhere since they belong to $L^{p_{0}}$.

Let $N>0$ and define $b_{N}$ as follows:

$$
b_{N}(x)= \begin{cases}-N, & b(x)<-N, \\ b(x), & -N \leq b(x) \leq N, \\ N, & b(x)>N .\end{cases}
$$

Then, it is immediate to see that $\left|b_{N}(x)-b_{N}(y)\right| \leq|b(x)-b(y)|$ for all $x, y$. Thus, $\left\|b_{N}\right\|_{\dot{\Lambda}_{\beta}} \leq\|b\|_{\dot{\Lambda}_{\beta}}$. As $b_{N} \in L^{\infty}$, we can use (33) and

$$
\begin{aligned}
\left\|\left[b_{N}, T\right] f\right\|_{L^{q}(v)} & \leq C\left\|b_{N}\right\|_{\dot{\Lambda}_{\beta}}\|f\|_{L^{p}(w)} \\
& \leq C\|b\|_{\dot{\Lambda}_{\beta}}\|f\|_{L^{p}(w)}<\infty .
\end{aligned}
$$

To conclude, by Fatou's lemma, it suffices to show that $\left|\left[b_{N_{j}}, T\right] f(x)\right| \rightarrow|[b, T] f(x)|$ for a.e. $x \in \mathbb{R}^{n}$ and for some subsequence $\left\{N_{j}\right\}_{j}$ such that $N_{j} \rightarrow \infty$.

As $\left|b_{N}\right| \leq|b| \in L^{p}\left(Q_{0}\right)$, for any $1 \leq p<\infty$, the dominated convergence theorem yields that $b_{N} f \rightarrow b f$ in $L^{p_{0}}$ as $N \rightarrow$ $\infty$. Therefore, $T$ is bounded from $L^{p_{0}}$ to $L^{s_{0}}$. It follows that $T\left(b_{N} f-b f\right) \rightarrow 0$ in $L^{s_{0}}$. Thus, there exists a subsequence $N_{j} \rightarrow \infty$ such that $T\left(b_{N_{j}} f-b f\right) \rightarrow 0$ for a.e. $x \in \mathbb{R}^{n}$. In this way we obtain

$$
\begin{aligned}
& \|\left[b_{N_{j}}, T\right] f(x)|-|[b, T] f(x)|| \\
& \quad \leq\left|\left[b_{N_{j}}, T\right] f(x)-[b, T] f(x)\right| \\
& \quad \leq\left|T\left(b_{N_{j}} f-b f\right)(x)\right|+\left|b_{N_{j}}(x)-b(x)\right||T f(x)|
\end{aligned}
$$

as desired, and we get that $\left|\left[b_{N_{j}}, T\right] f(x)\right| \rightarrow|[b, T] f(x)|$ for a.e $x \in \mathbb{R}^{n}$.

Proof of Theorem 1. We assume that $q_{0}<\infty$, for $q_{0}=\infty$, and the main ideas are the same and details are left to the interested reader. Lemma 9 ensures that it suffices to consider the case $b \in \dot{\Lambda}_{\beta} \cap L^{\infty}$. Let $f \in L_{c}^{\infty}$ and set $F=|[b, T] f|^{s_{0}}$. Note that $F \in L^{1}$ by (i) of Lemma 9. Given a ball $B$, we set $f_{B, b}=\left(b_{4 B}-b\right) f$ and decompose $[b, T] f$ as follows:

$$
\begin{aligned}
\mid[b, T] & f(x) \mid \\
= & |T((b(x)-b) f)(x)| \\
\leq & \left|b(x)-b_{4 B}\right||T f(x)|+\left|T\left(\left(b_{4 B}-b\right) f\right)(x)\right| \\
\leq & \left|b(x)-b_{4 B}\right||T f(x)|+\left|T\left(I-A_{r(B)}\right) f_{B, b}(x)\right| \\
& +\left|T A_{r(B)} f_{B, b}(x)\right| .
\end{aligned}
$$


We observe that $F \leq G_{B}+H_{B}$, where

$$
\begin{aligned}
G_{B} & =4^{s_{0}-1}\left(G_{B, 1}+G_{B, 2}\right) \\
& =4^{s_{0}-1}\left(\left|b-b_{4 B}\right|^{s_{0}}|T f|^{s_{0}}+\left|T\left(I-A_{r(B)}\right) f_{B, b}\right|^{s_{0}}\right)
\end{aligned}
$$

and $H_{B}=2^{s_{0}-1}\left|T A_{r(B)} f_{B, b}\right|^{s_{0}}$.

We first estimate the average of $G_{B}$ on $B$. Fix any $x \in B$. Let $1<s<\infty$. Using Lemma 4 ,

$$
\begin{aligned}
\left(f_{B} G_{B, 1}\right)^{1 / s_{0}}= & \left(\frac{1}{|B|} \int_{B}\left|b-b_{4 B}\right|^{s_{0}}|T f|^{s_{0}}\right)^{1 / s_{0}} \\
\leq & \left(\frac{1}{|B|} \int_{B}\left|b-b_{4 B}\right|^{s_{0} s^{\prime}}\right)^{1 /\left(s_{0} s^{\prime}\right)} \\
& \times\left(\frac{1}{|B|} \int_{B}|T f|^{s_{0} s}\right)^{1 /\left(s_{0} s\right)} \\
= & \frac{1}{|B|^{\beta / n}}\left(\frac{1}{|B|} \int_{B}\left|b-b_{4 B}\right|^{s_{0} s^{\prime}}\right)^{1 /\left(s_{0} s^{\prime}\right)} \\
& \times\left(\frac{1}{|B|^{1-s_{0} s \beta / n}} \int_{B}|T f|^{s_{0} s}\right)^{1 /\left(s_{0} s\right)} \\
\leq & C\|b\|_{\dot{\Lambda}_{\beta}} M_{\beta, s_{0} s}(T f)(x) .
\end{aligned}
$$

Using (9) and Lemmas 4 and 5,

$$
\begin{aligned}
\left(f_{B} G_{B, 2}\right)^{1 / s_{0}}= & \left(f_{B}\left|T\left(I-A_{r(B)}\right) f_{B, b}\right|^{s_{0}}\right)^{1 / s_{0}} \\
\leq & C \sum_{j=1}^{\infty} \alpha_{j}\left|2^{j+1} B\right|^{\alpha / n}\left(f_{2^{j+1} B}\left|f_{B, b}\right|^{p_{0}}\right)^{1 / p_{0}} \\
\leq & \left.\left.C \sum_{j=1}^{\infty} \alpha_{j}\right|^{j+1} B\right|^{\alpha / n} \\
& \times\left(\frac{1}{\left|2^{j+1} B\right|} \int_{2^{j+1} B}^{\left.\left|b-b_{2^{j+1} B}\right|^{p_{0}}|f|^{p_{0}}\right)^{1 / p_{0}}}\right. \\
& +C \sum_{j=1}^{\infty} \alpha_{j}\left|2^{j+1} B\right|^{\alpha / n} \\
& \times\left(\frac{1}{\left|2^{j+1} B\right|}\left|b_{2^{j+1} B}-b_{4 B}\right|^{p_{0}} \int_{2^{j+1} B}|f|^{p_{0}}\right)^{1 / p_{0}} \\
\leq & C \sum_{j=1}^{\infty} \alpha_{j}\|b\|_{\Lambda_{\beta}} M_{\alpha+\beta, p_{0} s}(f)(x) \\
& +C \sum_{j=1}^{\infty} \alpha_{j}\|b\|_{\Lambda_{\beta}}\left|2^{j+1} B\right|^{(\alpha+\beta) / n}
\end{aligned}
$$

$$
\begin{aligned}
& \times\left(\frac{1}{\left|2^{j+1} B\right|} \int_{2^{j+1} B}|f|^{p_{0} s}\right)^{1 /\left(p_{0} s\right)} \\
\leq & C\|b\|_{\dot{\Lambda}_{\beta}} M_{\alpha+\beta, p_{0} s}(f)(x)
\end{aligned}
$$

since $\sum_{j=1}^{\infty} \alpha_{j}<\infty$. Hence, for any $x \in B$,

$$
\begin{aligned}
f_{B} G_{B} \leq C\left(\|b\|_{\dot{\Lambda}_{\beta}}^{s_{0}} M_{\beta, s_{0} s}(T f)^{s_{0}}(x)\right. \\
\left.\quad+\|b\|_{\dot{\Lambda}_{\beta}}^{s_{0}} M_{\alpha+\beta, p_{0} s}(f)^{s_{0}}(x)\right) \equiv G(x) .
\end{aligned}
$$

We next estimate the average of $H_{B}^{q^{\prime}}$ on $B$ with $q^{\prime}=q_{0} / s_{0}$. Using (10) and proceeding as before, we see that

$$
\begin{aligned}
\left(f_{B} H_{B}^{q^{\prime}}\right)^{1 / q_{0}} & 2^{\left(s_{0}-1\right) / s_{0}}\left(f_{B}\left|T A_{r(B)} f_{B, b}\right|^{q_{0}}\right)^{1 / q_{0}} \\
\leq & C \sum_{j=1}^{\infty} \alpha_{j}\left(f_{2^{j+1} B}\left|T f_{B, b}\right|^{s_{0}}\right)^{1 / s_{0}} \\
\leq & C \sum_{j=1}^{\infty} \alpha_{j}\left(f_{2^{j+1} B}\left|T_{b} f\right|^{s_{0}}\right)^{1 / s_{0}} \\
& +C \sum_{j=1}^{\infty} \alpha_{j}\left(f_{2^{j+1} B}\left|b-b_{4 B}\right|^{s_{0}}|T f|^{s_{0}}\right)^{1 / s_{0}} \\
\leq & C(M F)^{1 / s_{0}}(x)+C\|b\|_{\dot{\Lambda}_{\beta}} M_{\beta, s_{0} s}(T f)(\bar{x}),
\end{aligned}
$$

for any $x, \bar{x} \in B$. Thus we have obtained

$$
\begin{aligned}
\left(f_{B} H_{B}^{q^{\prime}}\right)^{1 / q^{\prime}} & \leq C\left(M F(x)+\|b\|_{\dot{\Lambda}_{\beta}}^{s_{0}} M_{\beta, s_{0} s}(T f)^{s_{0}}(\bar{x})\right) \\
& \equiv C\left(M F(x)+H_{2}(\bar{x})\right) .
\end{aligned}
$$

For $p_{0}<p<q<q_{0}$ and $1 / q=1 / p-(\alpha+\beta) / n$, we can find a $1<s<\infty$ such that $s_{0} s<1 /(1 / p-\alpha / n)$ and $p_{0} s<p$. As mentioned before $F \in L^{1}$. Applying Theorem A and Remark 2 with $q / s_{0}$ in place of $p$, we obtain

$\|[b, T] f\|_{q}^{s_{0}}$

$$
\begin{aligned}
& \leq\|M F\|_{q / s_{0}} \leq C\left(\|G\|_{q / s_{0}}+\left\|H_{2}\right\|_{q / s_{0}}\right) \\
& \leq C\|b\|_{\dot{\Lambda}_{\beta}}^{s_{0}}\left(\left\|M_{\beta, s_{0} s}(T f)\right\|_{q}^{s_{0}}+\left\|M_{\alpha+\beta, p_{0} s}(f)\right\|_{q}^{s_{0}}\right) \\
& \leq C\|b\|_{\dot{\Lambda}_{\beta}}^{s_{0}}\left(\|T f\|_{1 /(1 / p-\alpha / n)}^{s_{0}}+\|f\|_{p}^{s_{0}}\right) \\
& \leq C\|b\|_{\dot{\Lambda}_{\beta}}^{s_{0}}\|f\|_{p}^{s_{0}},
\end{aligned}
$$


where we have used Lemma 6. This implies that

$$
\|[b, T] f\|_{q} \leq C\|b\|_{\dot{\Lambda}_{\beta}}\|f\|_{p} .
$$

Proof of Theorem 3. Let $F, G$, and $H_{2}$ be the same as those used in the proof of Theorem 1 . As mentioned before $F \in L^{1}$. Since $v \in A_{p / p_{0}} \cap R H_{\left(q_{0} / p\right)^{\prime}}$, applying Theorem A with $p / p_{0}$ in place of $p$ and $s=q_{0} / p$, we obtain

$$
\begin{gathered}
\|[b, T] f\|_{L^{q}(v)}^{p_{0}} \\
\leq\|M F\|_{L^{q / p_{0}(v)}} \leq C\left(\|G\|_{L^{q / p_{0}}(v)}+\left\|H_{2}\right\|_{L^{q / p_{0}}(v)}\right) \\
\leq C\|b\|_{\dot{\Lambda}_{\beta}}^{p_{0}}\left(\left\|M_{\beta, p_{0} s}(T f)\right\|_{L^{q}(v)}^{p_{0}}+\left\|M_{\beta, p_{0} s}(f)\right\|_{L^{q}(v)}^{p_{0}}\right) \\
=C\|b\|_{\dot{\Lambda}_{\beta}}^{p_{0}}\left(\left\|M_{\beta p_{0} s / n, p_{0} s}(T f)\right\|_{L^{q}(v)}^{p_{0}}\right. \\
\left.+\left\|M_{\beta p_{0} s / n, p_{0} s}(f)\right\|_{L^{q}(v)}^{p_{0}}\right) .
\end{gathered}
$$

Noting that $(w, v) \in A\left(p / p_{0} s, q / p_{0} s, \beta p_{0} s / n\right)$, Lemma 8 and Remark 2 give us that

$$
\begin{aligned}
\left\|\mathscr{M}_{\beta p_{0} s / n, p_{0} s}(T f)\right\|_{L^{q}(v)} & \leq C\|T f\|_{L^{p}(w)} \\
& \leq C\|f\|_{L^{p}(w)} .
\end{aligned}
$$

This implies that

$$
\|[b, T] f\|_{L^{q}(v)} \leq C\|b\|_{\dot{\Lambda}_{\beta}}\|f\|_{L^{p}(w)} .
$$

\section{Applications}

4.1. Spectral Multipliers: Off-Diagonal Estimates. Suppose that $L$ is a self-adjoint nonnegative definite operator on $L^{2}\left(\mathbb{R}^{n}\right)$. Let $E(\lambda)$ be the spectral resolution of $L$. For any bounded Borel function $m:[0, \infty) \rightarrow \mathbb{C}$, by using the spectral theorem, we can define the operator

$$
m(L)=\int_{0}^{\infty} m(\lambda) d E(\lambda) .
$$

This is of course bounded on $L^{2}\left(\mathbb{R}^{n}\right)$.

The following will be assumed throughout this subsection.

(H1) $L$ is a nonnegative self-adjoint operator on $L^{2}\left(\mathbb{R}^{n}\right)$.

(H2) The operator $L$ generates an analytic semigroup $\left\{e^{-t L}\right\}_{t>0}$ which satisfies the Davies-Gaffney condition. That is, there exist constants $C, c>0$ such that for any open subsets $U_{1}, U_{2} \subset \mathbb{R}^{n}$,

$$
\begin{aligned}
&\left|\left\langle e^{-t L} f_{1}, f_{2}\right\rangle\right| \\
& \leq C \exp \left(-\frac{\operatorname{dist}\left(U_{1}, U_{2}\right)^{2}}{c t}\right) \\
& \quad \times\left\|f_{1}\right\|_{L^{2}\left(\mathbb{R}^{n}\right)}\left\|f_{2}\right\|_{L^{2}\left(\mathbb{R}^{n}\right)}, \quad \forall t>0,
\end{aligned}
$$

for every $f_{i} \in L^{2}\left(\mathbb{R}^{n}\right)$ with supp $f_{i} \subset U_{i}, i=1,2$, where $\operatorname{dist}\left(U_{1}, U_{2}\right):=\inf _{x \in U_{1}, y \in U_{2}} d(x, y)$.

(H3) Suppose $2<q_{0} \leq \infty$. Assume that the analytic semigroup $e^{-t L}$ generated by $L$ satisfies " $L^{2}-L^{q_{0}}$ off-diagonal" estimates: there exist coefficients $\left\{a_{j}\right\}_{j \geq 0}$ satisfying $\sum_{j=0}^{\infty} a_{j}<\infty$ such that for all balls $B$ and for all functions $f \in L^{2}\left(\mathbb{R}^{n}\right)$

$$
\begin{aligned}
& \left(\frac{1}{|B|} \int_{B}\left|e^{-r_{B}^{2} L} f\right|^{q_{0}} d x\right)^{1 / q_{0}} \\
& \quad \leq \sum_{j=0}^{\infty} a_{j}\left(\frac{1}{\left|2^{j} B\right|} \int_{2^{j} B}|f|^{2} d x\right)^{1 / 2} .
\end{aligned}
$$

Let $\phi$ be a nonnegative $C_{0}^{\infty}$ function such that

$$
\operatorname{supp} \phi \subset\left(\frac{1}{4}, 1\right), \quad \sum_{l \in \mathbb{Z}} \phi\left(2^{-l} \lambda\right)=1, \quad \forall \lambda>0 .
$$

For $s \geq 0$, let $[s]$ denote the integer part of $s$. Recall that $C^{s}$ is the space of functions $m$ on $\mathbb{R}$ for which

$\|m\|_{C^{s}}$

$$
= \begin{cases}\sum_{k=0}^{s} \sup _{\lambda \in \mathbb{R}}\left|m^{(k)}(\lambda)\right| & \text { if } s \in \mathbb{Z}, \\ \left\|m^{([s])}\right\|_{\operatorname{Lip}(s-[s])}+\sum_{k=0}^{[s]} \sup _{\lambda \in \mathbb{R}}\left|m^{(k)}(\lambda)\right| & \text { if } s \notin \mathbb{Z}\end{cases}
$$

is finite.

Then the following result holds.

Theorem 10. Let L satisfy assumptions (H1)-(H3). Let $\phi$ be a nonnegative $C_{0}^{\infty}$ function satisfying (54), and suppose that the bounded measurable function $m:[0, \infty) \rightarrow \mathbb{C}$ satisfies

$$
C_{\phi, s}=\sup _{t>0}\|\phi(\cdot) m(t \cdot)\|_{C^{s}}+|m(0)|<\infty
$$

for some $s>n / 2$. Then

(i) let $0<\beta<1$. If $2<p<1 /\left(1 / q_{0}+\beta / n\right)$ and $1 / q=$ $1 / p-\beta / n$, then there is a constant $C$ such that

$$
\|[b, m(L)] f\|_{L^{q}} \leq C\|b\|_{\dot{\Lambda}_{\beta}}\|f\|_{L^{p}},
$$

for all $f \in L_{c}^{\infty}$ and for all $b \in \dot{\Lambda}_{\beta}$.

(ii) Let $0<\beta<\min \{1, n / 2\}, 2<p<q<q_{0}$, and $w, v \in A_{p / p_{0}} \cap R H_{\left(q_{0} / p\right)^{\prime}}$. If there exists $a$ constant $1<s<\min \{n / \beta 2, p / 2\}$ such that $(w, v) \in$ $A(p / 2 s, q / 2 s, \beta 2 s / n)$, then there is a constant $C$ such that

$$
\|[b, m(L)] f\|_{L^{q}(v)} \leq C\|b\|_{\dot{\Lambda}_{\beta}}\|f\|_{L^{p}(w)},
$$

for all $f \in L_{c}^{\infty}$ and for all $b \in \dot{\Lambda}_{\beta}$. 
Proof. Estimate (57) follows from Theorem 1 with $\alpha=0$ and estimate (58) follows from Theorem 3, applied to $T f=m(L) f$ and $A_{r}=I-\left(I-e^{-r^{2} L}\right)^{M}$ with $M \in \mathbb{N}$ and $M>s / 2$. It suffices to show that there exist coefficients $\left\{a_{j}\right\}_{j \geq 0}$ satisfying $\sum_{j=1}^{\infty} a_{j}<\infty$ such that (9) and (10) hold for all $f \in L_{c}^{\infty}\left(\mathbb{R}^{n}\right)$.

Fix $1 \leq k \leq M$. From (53), we deduce that

$$
\begin{aligned}
& \left(\frac{1}{|B|} \int_{B}\left|e^{-k r_{B}^{2} L} f\right|^{q_{0}} d x\right)^{1 / q_{0}} \\
& \quad \leq \sum_{j=0}^{\infty} C a_{j}\left(\frac{1}{\left|2^{j} B\right|} \int_{2^{j} B}|f|^{2} d x\right)^{1 / 2} .
\end{aligned}
$$

This estimate with $m(L) f$ in place of $f$ yields (10). Since, by functional calculus, $m(L) e^{-k r^{2} L} f=e^{-k r^{2} L} m(L) f$, (9) was proved in [13].

4.2. Riesz Transforms. Let $A$ be an $n \times n$ matrix of complex and $L^{\infty}$-valued coefficients on $\mathbb{R}^{n}$. We assume that this matrix satisfies the following ellipticity (or "accretivity") condition: there exist $0<\lambda \leq \Lambda<\infty$ such that

$$
\lambda|\xi|^{2} \leq \operatorname{Re} A(x) \xi \cdot \bar{\xi}, \quad|A(x) \xi \cdot \bar{\zeta}| \leq \Lambda|\xi||\zeta|
$$

for all $\xi, \zeta \in \mathbb{C}^{n}$ and almost every $x \in \mathbb{R}^{n}$. Associated with this matrix we define the second-order divergence form operator

$$
L=-\operatorname{div}(A \nabla)
$$

The Riesz transforms associated to $L$ are $\partial_{j} L^{-1 / 2}, 1 \leq$ $j \leq n$. Set $\nabla L^{-1 / 2}=\left(\partial_{1} L^{-1 / 2}, \ldots, \partial_{n} L^{-1 / 2}\right)$. The solution of the Kato conjecture [14] implies that this operator extends boundedly to $L^{2}$. This allows the representation

$$
\nabla L^{-1 / 2} f=\frac{1}{\sqrt{\pi}} \int_{0}^{\infty} \nabla e^{-t L} f \frac{d t}{\sqrt{t}}
$$

in which the integral converges strongly in $L^{2}$ both at 0 and $\infty$ when $f \in L^{2}$.

Define $\vartheta \in[0, \pi / 2)$ by

$$
\vartheta=\sup \{|\arg \langle L f, f\rangle|: f \in \mathscr{D}(L)\} \text {. }
$$

We write for $0<\theta<\infty, \Sigma_{\theta}=\{z \in \mathbb{C} \backslash\{0\}:|\arg z|<\theta\}$.

We extract from [15] some definitions and results on unweighted off-diagonal estimates.

Definition 11. Let $1 \leq p \leq q \leq \infty$. One says that a family $\left\{T_{t}\right\}_{t>0}$ of sublinear operators satisfies $L^{p}-L^{q}$ full off-diagonal estimates, in short $T_{t} \in \mathscr{F}\left(L^{p}-L^{q}\right)$, if for some $c>0$, for all closed sets $E$ and $F$, all $f$, and all $t>0$, we have

$$
\begin{aligned}
& \left(\int_{F}\left|T_{t}\left(\chi_{E} f\right)\right|^{q} d x\right)^{1 / q} \\
& \quad \leq C t^{-(1 / 2)(n / p-n / q)} e^{-c d^{2}(E, F) / 2}\left(\int_{E}|f|^{p} d x\right)^{1 / p} .
\end{aligned}
$$

If $I$ is a subinterval of $[1, \infty]$, Int $I$ denotes the interior in $\mathbb{R}$ of $I \cap \mathbb{R}$.

Proposition 12 (see [15]). Fix $m \in \mathbb{N}$ and $0<\mu<\pi / 2-\vartheta$.

(a) There exists a nonempty maximal interval in $[1, \infty]$, denoted by $\mathscr{J}(L)$, such that if $p, q \in \mathscr{J}(L)$ with $p \leq q$, then $\left\{(z L)^{m} e^{-z L}\right\}_{z \in \Sigma_{\mu}}$ satisfies $L^{p}-L^{q}$ full off-diagonal estimates and is a bounded set in $\mathscr{L}\left(L^{p}\right)$.

(b) There exists a nonempty maximal interval in $[1, \infty]$, denoted by $\mathscr{K}(L)$, such that if $p, q \in \mathscr{K}(L)$ with $p \leq$ $q$, then $\left\{\sqrt{z} \nabla(z L)^{m} e^{-z L}\right\}_{z \in \Sigma_{\mu}}$ satisfies $L^{p}-L^{q}$ full offdiagonal estimates and is a bounded set in $\mathscr{L}\left(L^{p}\right)$.

(c) $\mathscr{K}(L) \subset \mathscr{J}(L)$ and, for $p<2$, we have $p \in \mathscr{K}(L)$ if and only if $p \in \mathscr{J}(L)$.

(d) Denote by $p_{-}(L), p_{+}(L)$ the lower and upper bounds of $\mathscr{J}(L)$ and by $q_{-}(L), q_{+}(L)$ those of $\mathscr{K}(L)$. We have $p_{-}(L)=q_{-}(L)$ and $\left(q_{-}(L)\right)^{*} \leq p_{+}(L)$. (We have set $q^{*}=(q n /(n-q))$, the Sobolev exponent of $q$ when $q<n$ and $q^{*}=\infty$, otherwise.)

(e) If $n=1, \mathscr{J}(L)=\mathscr{K}(L)=[1, \infty]$. If $n=2, \mathscr{J}(L)=$ $[1, \infty]$ and $\mathscr{K}(L) \supset\left[1, q_{+}(L)\right)$ with $q_{+}(L)>2$.

(f) If $n \geq 3, p_{-}(L)<2 n /(n+2), p_{+}(L)>2 n /(n-2)$, and $q_{+}(L)>2$.

Then for $q_{-}<p_{0}<q_{0}<q_{+}, T=\nabla L^{-1 / 2}$ satisfy (9) and (10) with $\alpha=0$ and $A_{r}=I-\left(I-e^{-r^{2} L}\right)^{M}$, where $M$ is a large enough integer. For the proof of this argument, see [15]. So Theorem 1 with $\alpha=0$ and Theorem 3 can be applied to $T=\nabla L^{-1 / 2}$.

4.3. Fractional Operators. Let $L=-\operatorname{div}(A \nabla)$. The fractional power of an elliptic operator $L$ on $R^{n}$ is given formally by

$$
L^{-\alpha / 2}=\frac{1}{\Gamma(\alpha / 2)} \int_{0}^{\infty} t^{\alpha / 2} e^{-t L} \frac{d t}{t}
$$

with $\alpha>0$. There exist $p_{-}=p_{-}(L)$ and $p_{+}=p_{+}(L), 1 \leq p_{-}<$ $2<p_{+} \leq \infty$ such that the semigroup $\left\{e^{-t L}\right\}_{t>0}$ is uniformly bounded on $L^{p}$ for every $p_{-}<p<p_{+}$(see Proposition 12). We have the following results.

Lemma 13 (see [9]). Let $p_{-}<p_{0}<s_{0}<q_{0}<p_{+}$so that $1 / p_{0}-1 / s_{0}=\alpha / n$. Fix a ball $B$ with radius $r$. For $f \in L_{c}^{\infty}$ and $M$ large enough, one has

$$
\begin{aligned}
& \left(f_{B}\left|L^{-\alpha / 2}\left(I-e^{-r^{2} L}\right)^{M} f\right|^{s_{0}}\right)^{1 / s_{0}} \\
& \quad \leq C \sum_{j=1}^{\infty} g_{1}(j)\left|2^{j+1} B\right|^{\alpha / n}\left(f_{2^{j+1} B}|f|^{p_{0}}\right)^{1 / p_{0}},
\end{aligned}
$$


and for $1 \leq l \leq M$

$$
\begin{aligned}
& \left(f_{B}\left|L^{-\alpha / 2} e^{-l r^{2} L} f\right|^{q_{0}}\right)^{1 / q_{0}} \\
& \quad \leq C \sum_{j=1}^{\infty} g_{2}(j)\left(f_{2^{j+1} B}\left|L^{-\alpha / 2} f\right|^{s_{0}}\right)^{1 / s_{0}},
\end{aligned}
$$

where $g_{j}=C 2^{-j\left(2 M-n / s_{0}\right)}$ and $g_{2}(j)=C e^{-c 4^{j}}$.

Theorem 14. Let $p_{-}<p<q<p_{+}, 0<\alpha, \beta, \alpha+\beta<1$, and $1 / q=1 / p-(\alpha+\beta) / n$. Given $b \in \dot{\Lambda}_{\beta}$, one has

$$
\left\|\left[b, L^{-\alpha / 2}\right] f\right\|_{q} \leq C\|b\|_{\dot{\Lambda}_{\beta}}\|f\|_{p} .
$$

Proof. We are going to apply Theorem 1 to the linear operator $T=L^{-\alpha / 2}$. We fix $p_{-}<p<q<p_{+}, \alpha$, and $\beta$ so that $1 / q=$ $1 / p-(\alpha+\beta) / n$. Then we can find $p_{0}, q_{0}, s_{0}$ such that $1 / p_{0}-$ $1 / s_{0}=\alpha / n, p_{-}<p_{0}<s_{0}<q_{0}<p_{+}$, and $p_{0}<p<q<q_{0}$. Notice that as $1 \leq p_{-}<p_{+} \leq \infty$, we have that $1<p_{0}<s_{0}<$ $q_{0}<\infty$. By Theorem 1.2 in [9], we know that $T=L^{-\alpha / 2}$ is bounded from $L^{p_{0}}$ to $L^{s_{0}}$.

We take $A_{r}=I-\left(I-e^{-r^{2} L}\right)^{m}$, where $m \geq 1$ is an integer to be chosen. We apply Lemma 13 . Note that (66) is (9). Also, (10) follows from (67) after expanding $A_{r}=I-\left(I-e^{-r^{2} L}\right)^{m}$. Then, we have that $\sum_{j \leq 1} g_{i}(j)$ for $i=1,2$ by choosing $2 m>$ $n / s_{0}$. Consequently applying Theorem 1 , we conclude that $\left\|\left[b, L^{-\alpha / 2}\right] f\right\|_{q} \leq C\|b\|_{\dot{\Lambda}_{\beta}}\|f\|_{p}$.

\section{Acknowledgments}

The authors would like to thank the referee for carefully reading the paper and for making several useful suggestions. This research was supported by Tianyuan Fund for Mathematics (Grant no. 11226100), Specialized Research Fund for the Doctoral Program of Higher Education (Grant no. 20124410120002), and SRF of Guangzhou Education Bureau (Grant no. 2012A088).

\section{References}

[1] R. R. Coifman, R. Rochberg, and G. Weiss, "Factorization theorems for Hardy spaces in several variables," Annals of Mathematics, vol. 103, no. 3, pp. 611-635, 1976.

[2] S. Janson, "Mean oscillation and commutators of singular integral operators," Arkiv för Matematik, vol. 16, no. 2, pp. 263270, 1978.

[3] M. Bramanti and M. C. Cerutti, "Commutators of singular integrals on homogeneous spaces," Bollettino dell'Unione Matematica Italiana, vol. 10, no. 4, pp. 843-883, 1996.

[4] X. T. Duong and L. X. Yan, "Commutators of BMO functions and singular integral operators with non-smooth kernels," Bulletin of the Australian Mathematical Society, vol. 67, no. 2, pp. 187-200, 2003.

[5] X. T. Duong and A. MacIntosh, "Singular integral operators with non-smooth kernels on irregular domains," Revista Matemática Iberoamericana, vol. 15, no. 2, pp. 233-265, 1999.
[6] G. Hu and D. Yang, "Maximal commutators of BMO functions and singular integral operators with non-smooth kernels on spaces of homogeneous type," Journal of Mathematical Analysis and Applications, vol. 354, no. 1, pp. 249-262, 2009.

[7] P. Auscher and J. M. Martell, "Weighted norm inequalities, off-diagonal estimates and elliptic operators. Part I: general operator theory and weights," Advances in Mathematics, vol. 212, no. 1, pp. 225-276, 2007.

[8] S. Blunck and P. C. Kunstmann, "Calderón-Zygmund theory for non-integral operators and the $H^{\infty}$ functional calculus," Revista Matemática Iberoamericana, vol. 19, no. 3, pp. 919-942, 2003.

[9] P. Auscher and J. M. Martell, "Weighted norm inequalities for fractional operators," Indiana University Mathematics Journal, vol. 57, no. 4, pp. 1845-1869, 2008.

[10] M. Paluszyński, "Characterization of the Besov spaces via the commutator operator of Coifman, Rochberg and Weiss," Indiana University Mathematics Journal, vol. 44, no. 1, pp. 1-18, 1995.

[11] S. Chanillo, "A note on commutators," Indiana University Mathematics Journal, vol. 31, no. 1, pp. 7-16, 1982.

[12] A. Gogatishvili and V. Kokilashvili, "Criteria of strong type two-weighted inequalities for fractional maximal functions," Georgian Mathematical Journal, vol. 3, no. 5, pp. 423-446, 1996.

[13] X. T. Duong and L. X. Yan, "Spectral multipliers for Hardy spaces associated to non-negative self-adjoint operators satisfying Davies-Gaffney estimates," Journal of the Mathematical Society of Japan, vol. 63, no. 1, pp. 295-319, 2011.

[14] P. Auscher, S. Hofmann, M. Lacey, A. McIntosh, and Ph. Tchamitchian, "The solution of the Kato square root problem for second order elliptic operators on $\mathbb{R}^{n}$," Annals of Mathematics, vol. 156, no. 2, pp. 633-654, 2002.

[15] P. Auscher and J. M. Martell, "Weighted norm inequalities, offdiagonal estimates and elliptic operators. Part III: harmonic analysis of elliptic operators "' Journal of Functional Analysis, vol. 241, no. 2, pp. 703-746, 2006. 


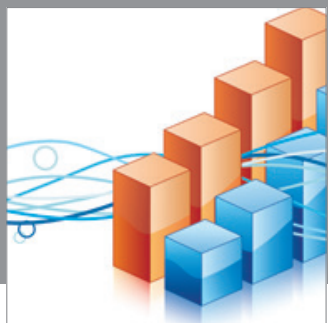

Advances in

Operations Research

mansans

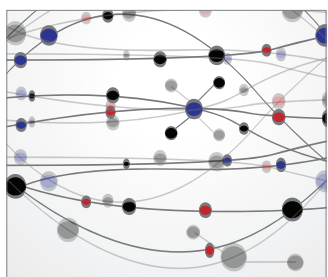

The Scientific World Journal
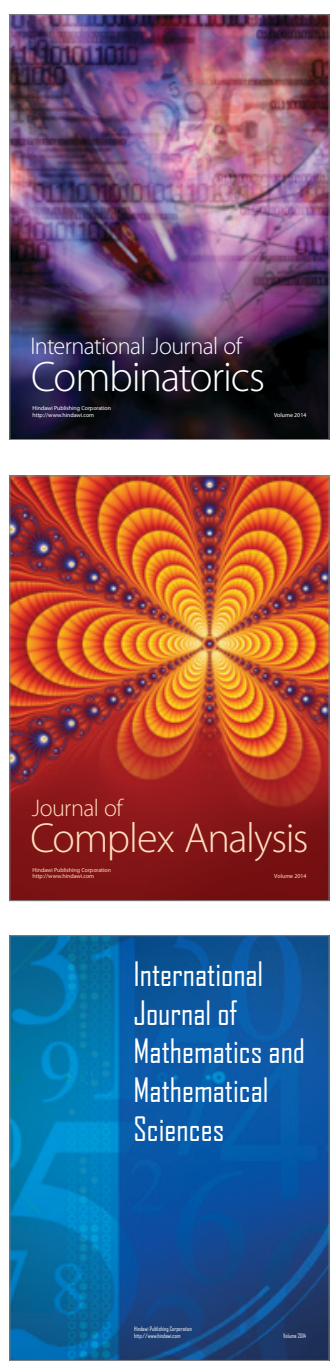
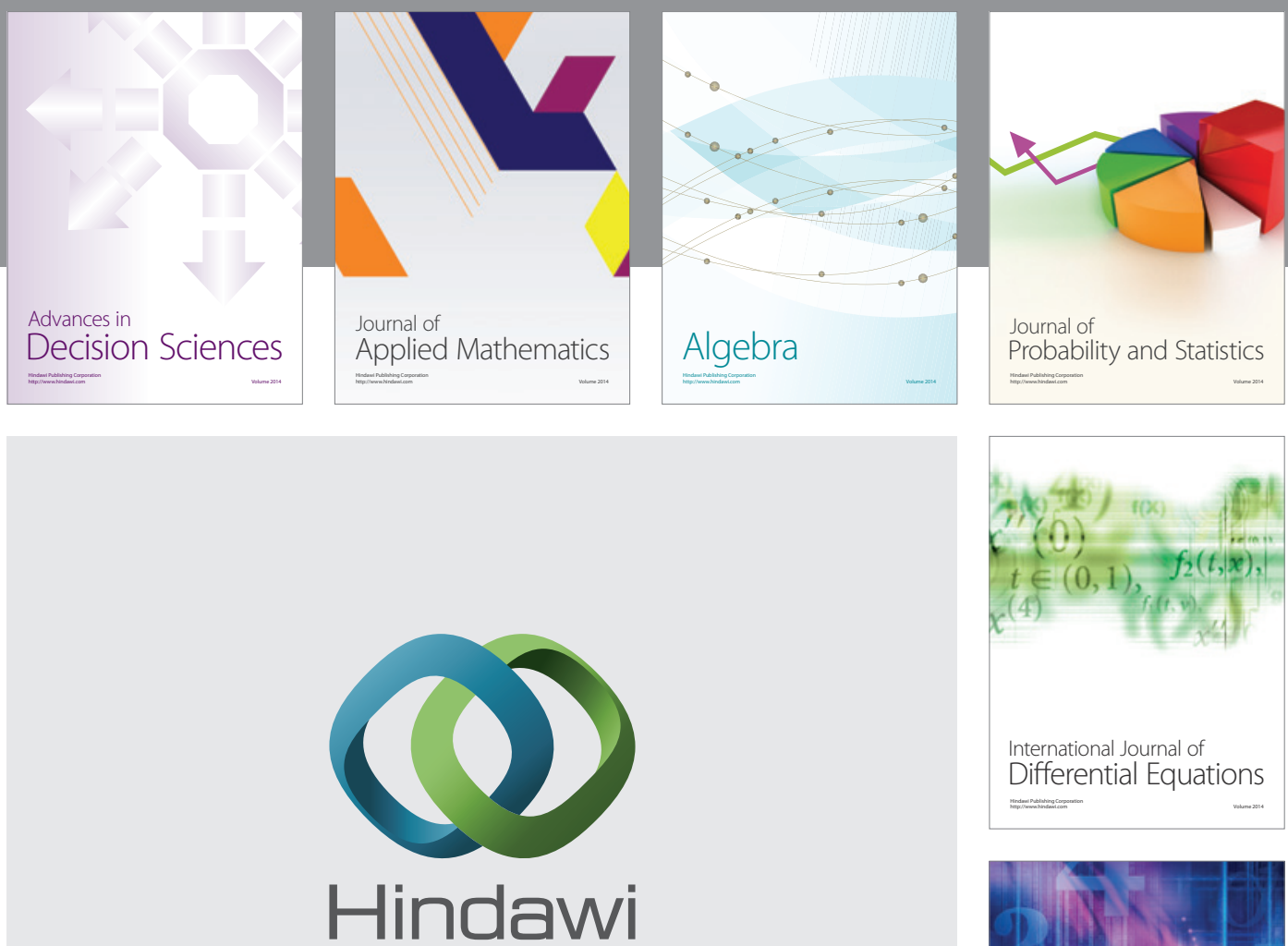

Submit your manuscripts at http://www.hindawi.com
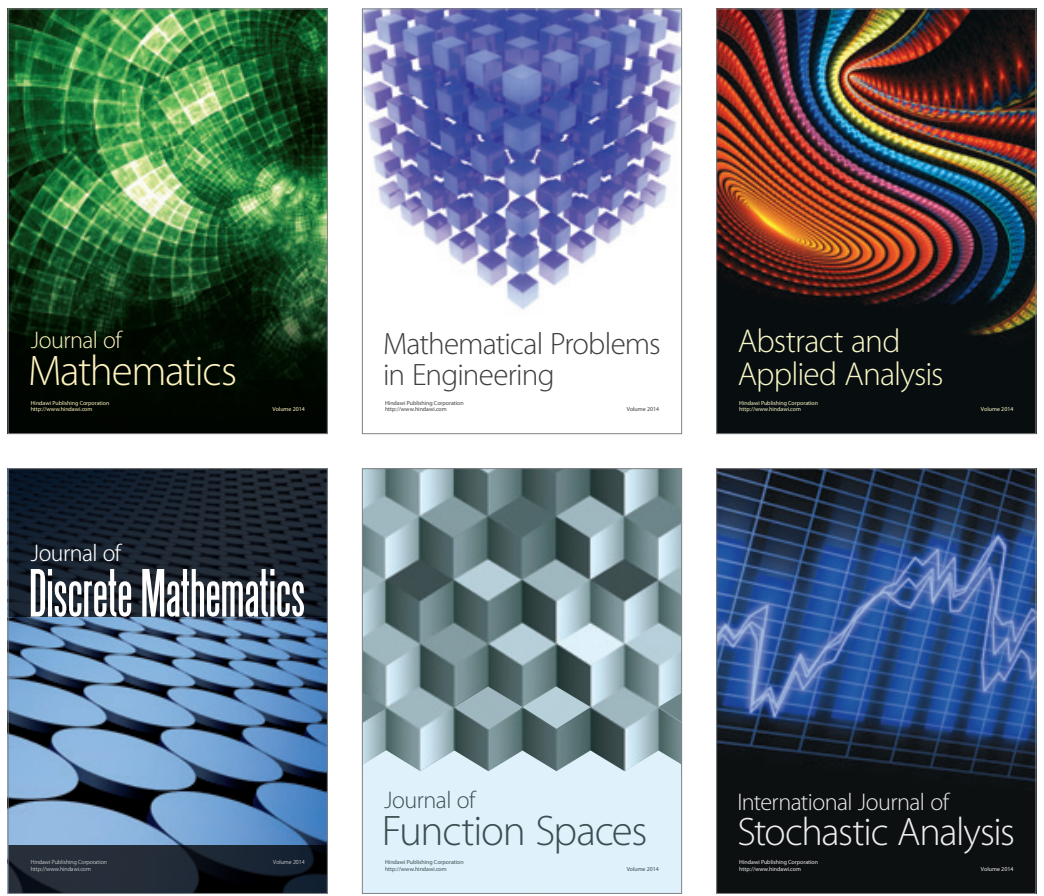

Journal of

Function Spaces

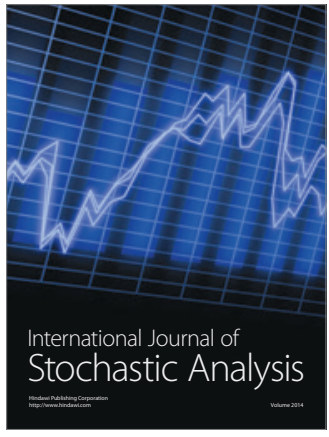

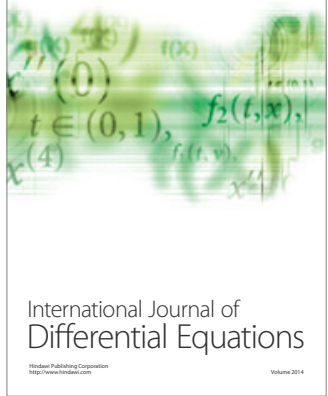
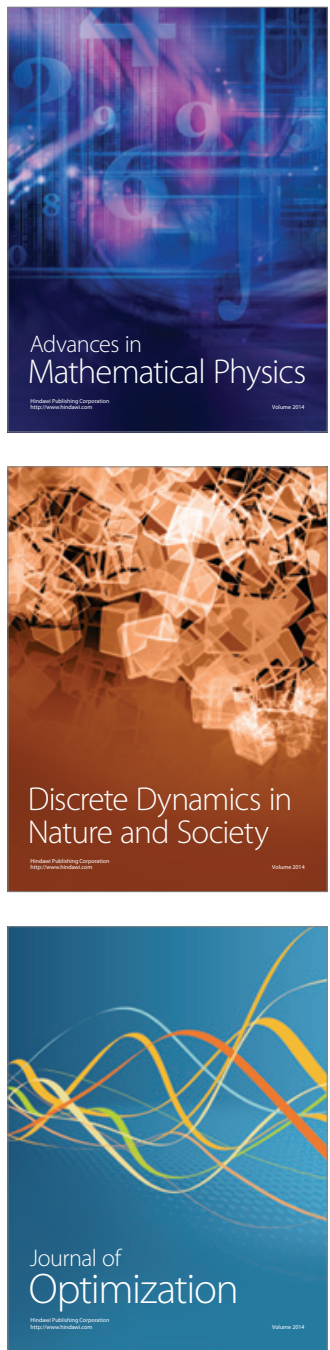